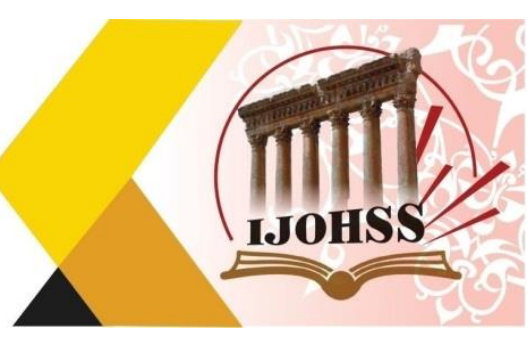

\title{
العلاقات الامريكية الاسبانية عقب انتهاء الحرب الاهلية الاسبانية
}

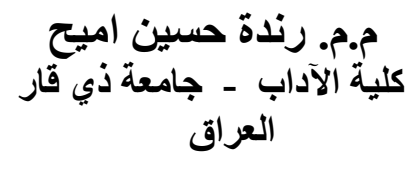

م.م. رندة حسين اميج

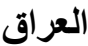

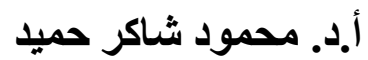

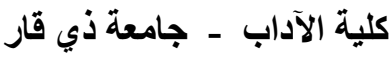

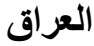

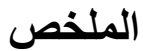

دخلت الولايات المتحدة الأمريكية بعلاقات جديدة مع إسبانيا عقب انتهاء الحرب الأهلية الاسبانية عام 1939،

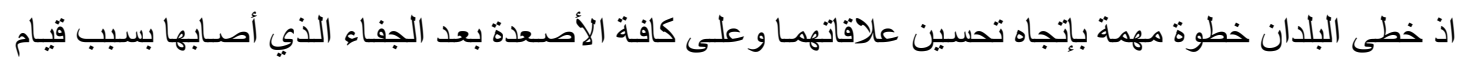

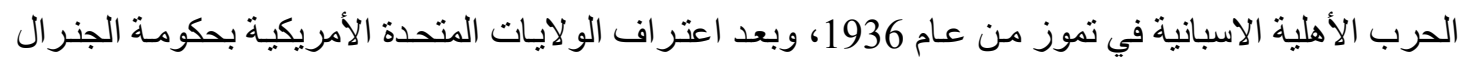
فر انكو عقد الجانبان العديد من الاتفاقيات الإستر اتيجية والتجارية والثقافية، تحسّنت على أثر هـا علاقات الجيات الجانبين

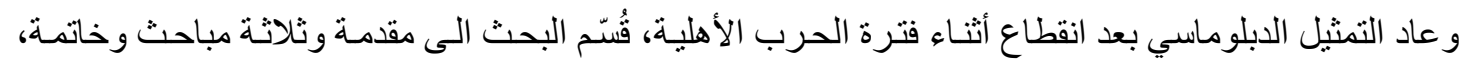
تكلم المبحث الأول عن الدبلوماسية الأمريكية ـ الاسبانية عقب انتهاء الحرب الأهلية الاسبانية، وناقش المبحث

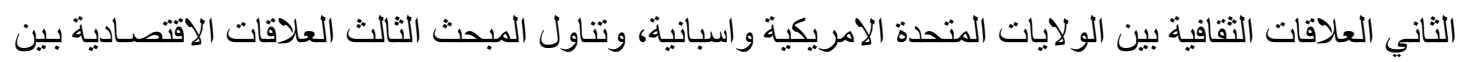
الو لايات المتحدة و اسبانيا ، واشتملت الخاتمة على اهم ما توصل إليه البحث من نتائج. 


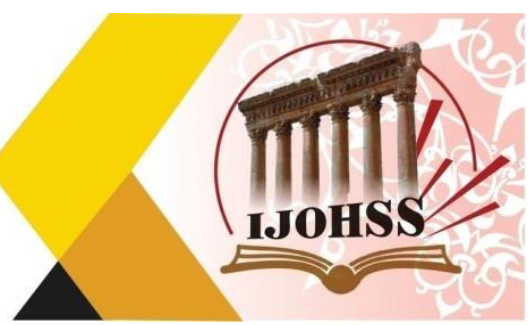

\title{
American-Spanish Relations after the End of the Spanish Civil War
}

\section{Prof. Dr. Mahmoud Shaker Hamid College of Arts - Dhi Qar University} Iraq

\author{
Assist. Prof.Eng. Randa Hussein Amih \\ College of Arts - Dhi Qar University \\ Iraq
}

\begin{abstract}
The United States of America entered into new relations with Spain after the end of the Spanish Civil War in 1939, as the two countries took an important step towards improving their relations at all levels after the estrangement that afflicted them due to the outbreak of the Spanish Civil War in July 1936, and after the United States recognized the government of General Franco. The two sides concluded many strategic, commercial and cultural agreements, as a result of which relations between the two sides improved and diplomatic representation returned after a break during the civil war period. The research was divided into an introduction, three topics and a conclusion. The first discussion spoke about US-Spanish diplomacy after the end of the Spanish Civil War, and the second topic discussed relations. Cultural relations between the United States of America and Spain. The third topic dealt with the economic relations between the United States and Spain. The conclusion included the most important findings of the research.
\end{abstract}

Keywords: American-Spanish Relations, Spanish Civil War. 


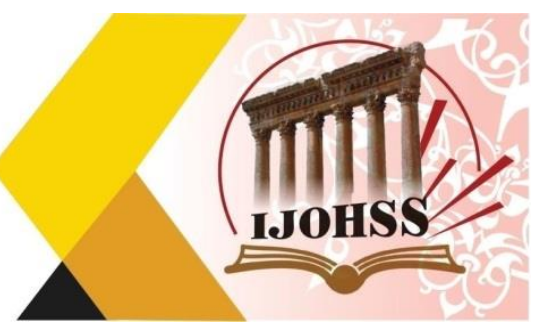

\section{المبحث الاول \\ الابلوماسية الامريكية ـ الاسبانية عقب انتهاء الحرب الاهلية الاسبانية}

كانت العلاقات الدبلوماسية والسياسية التى أبرمتها إسبانيا مع الو لايات المتحدة و واحدة قد بدأت تبرز على العيل السـاحة

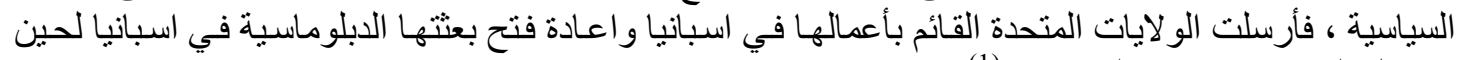

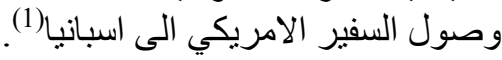

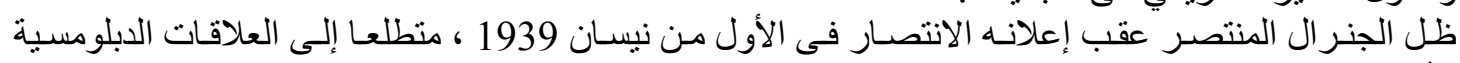

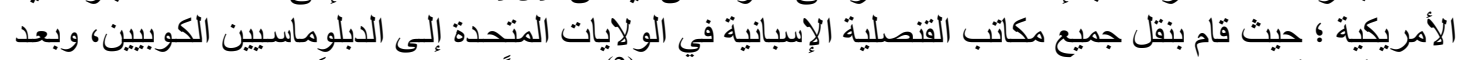

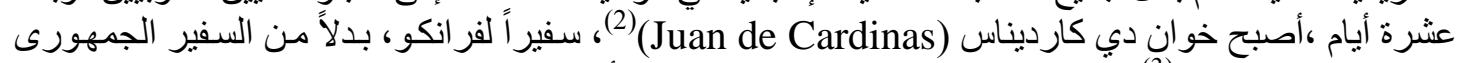

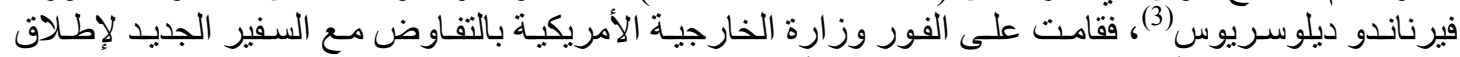

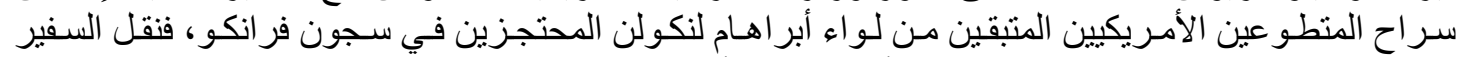

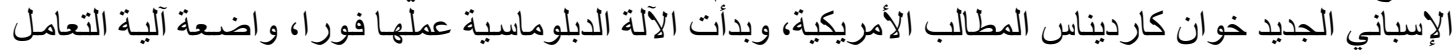

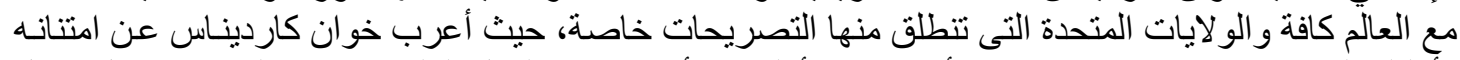

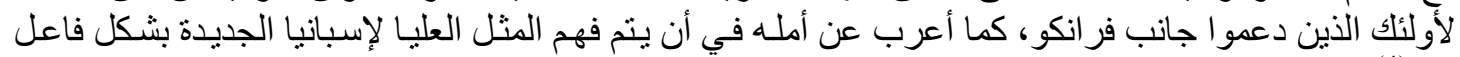

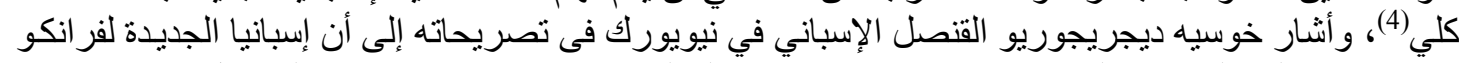

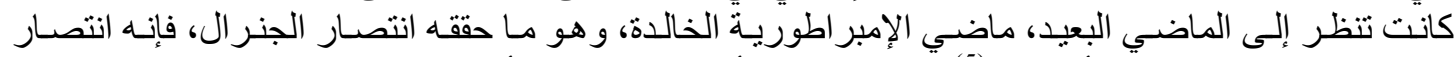

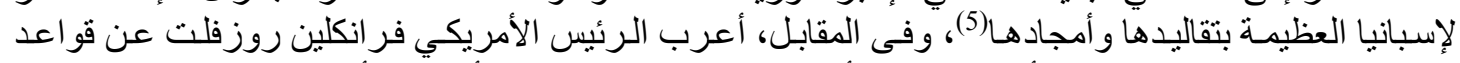

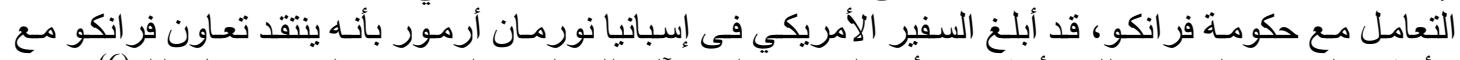

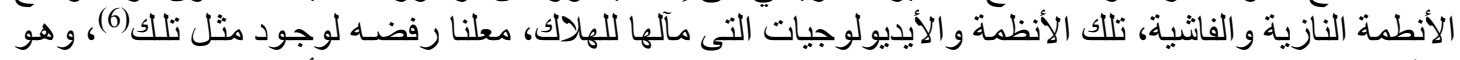

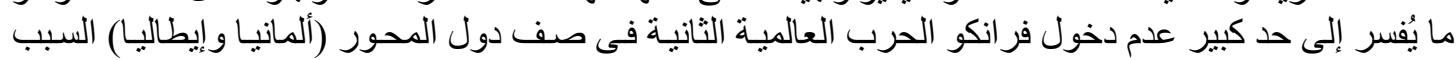

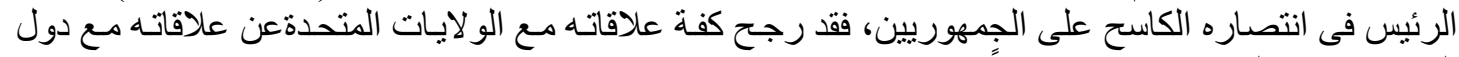

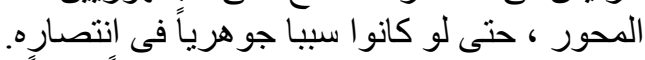

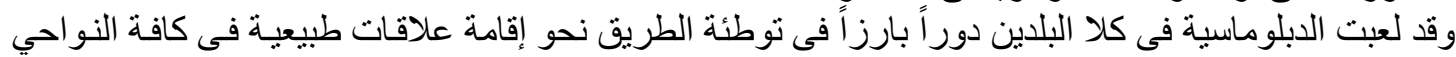

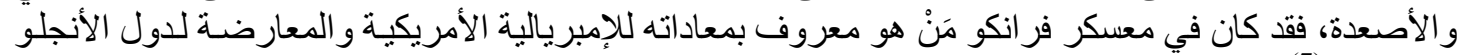

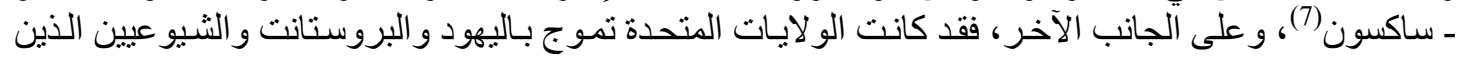

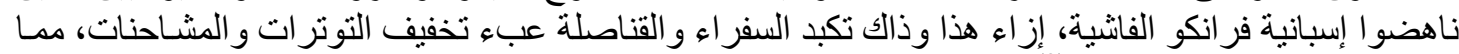

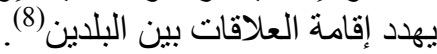

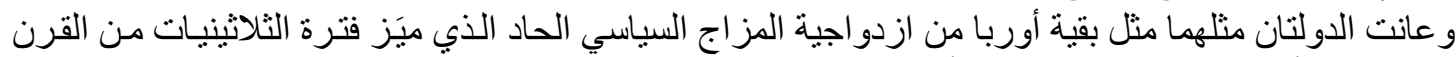

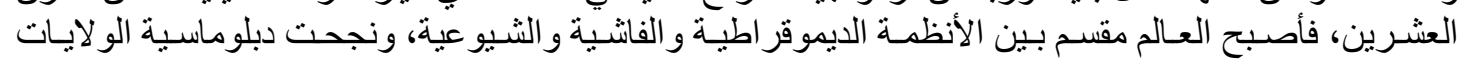

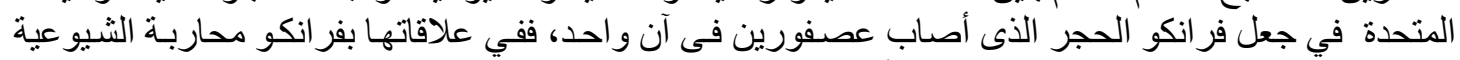

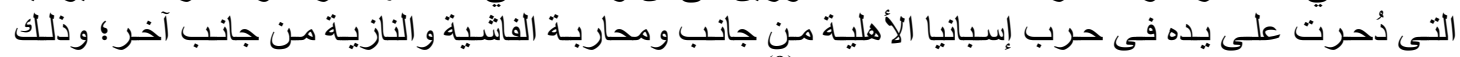

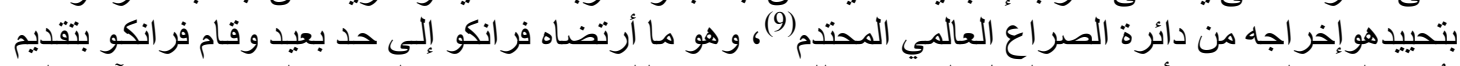

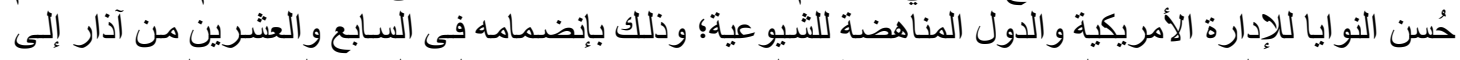

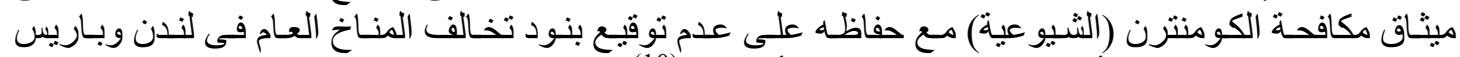

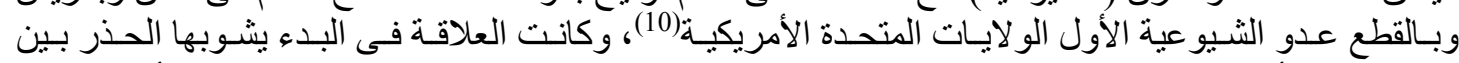

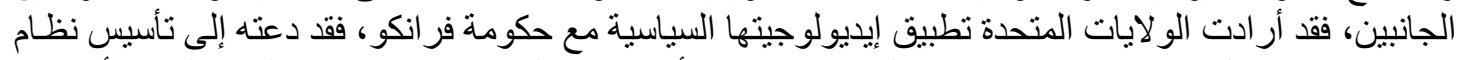

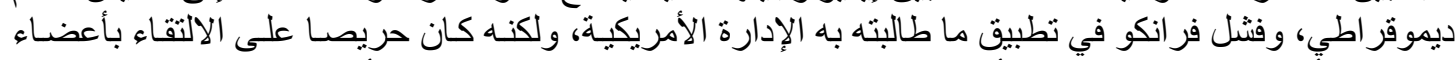

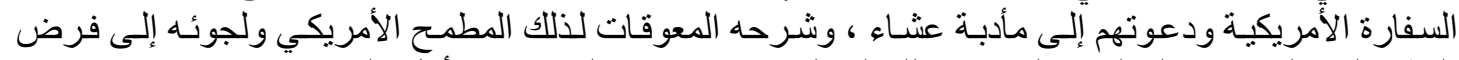

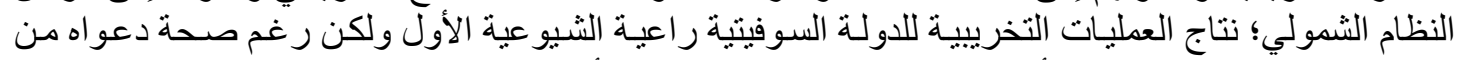

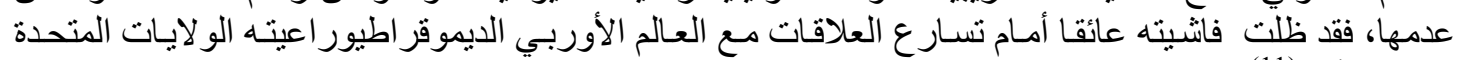
بالمقام الأول(11). (11).

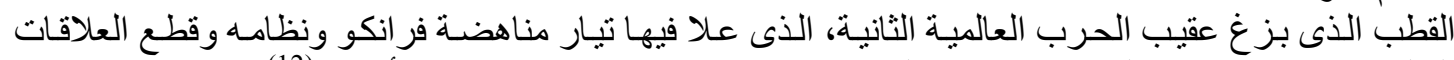

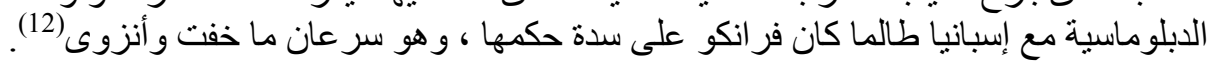




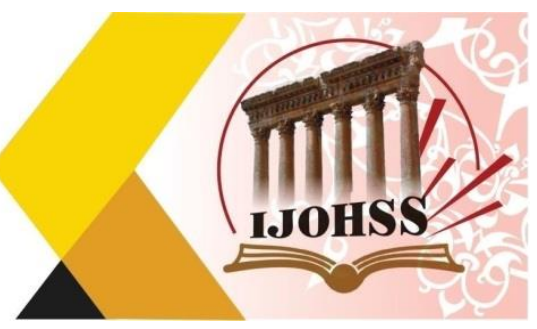

وقد كانت العلاقات الدبلوماسية والسياسية الأمريكية الإسبانية التى أستهلت عقيب انتهاء الحرب الكباء الأهلية الإسبانية

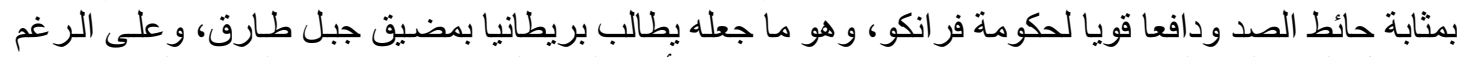

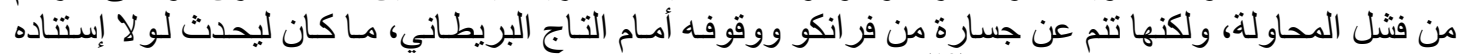

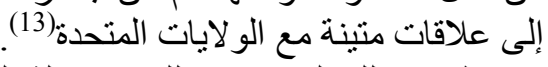

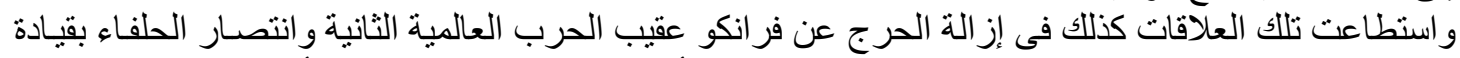

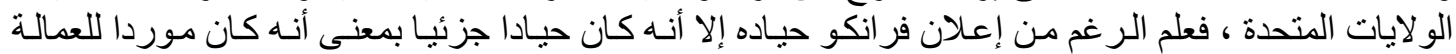

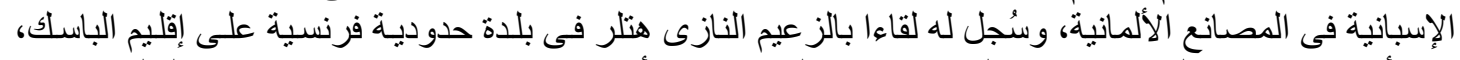

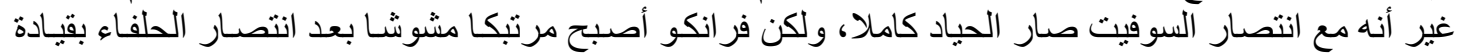

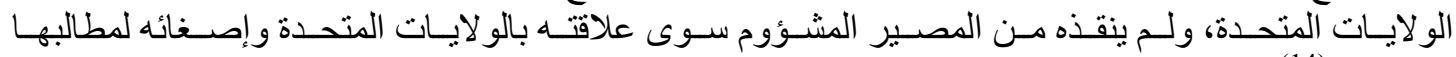

وشروطهان(14).

\section{العلاقات الثقافية بين الولايات المتحدة الأمريكية واسباتيا}

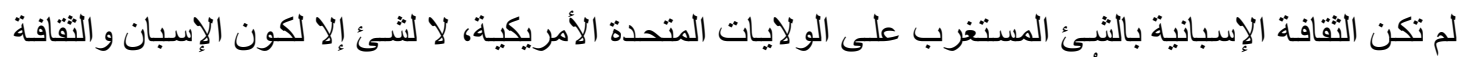

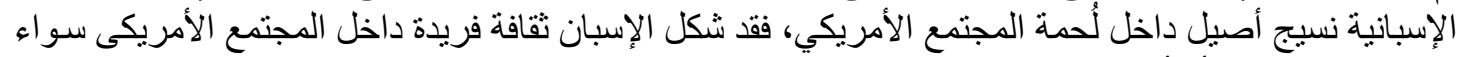

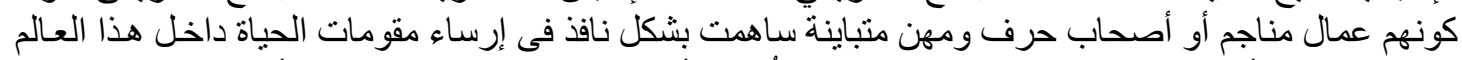

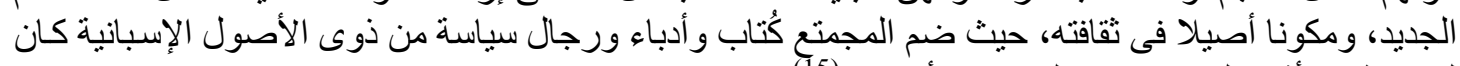

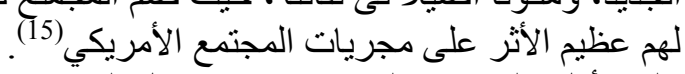

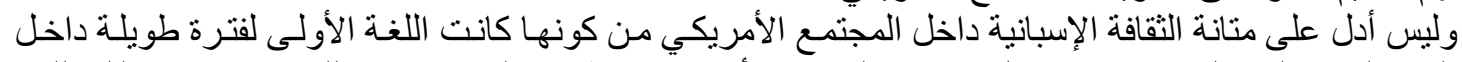





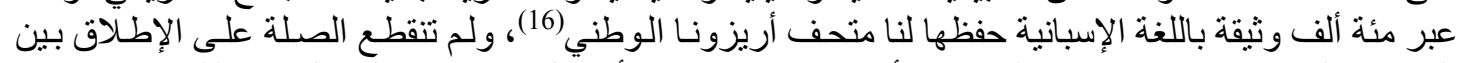

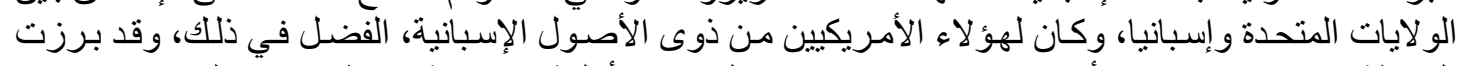

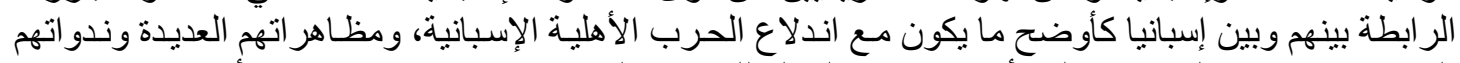

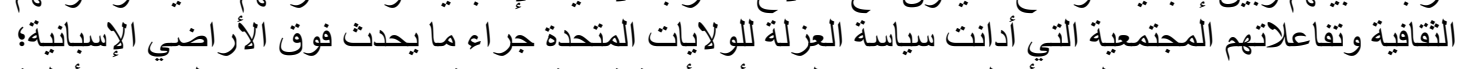

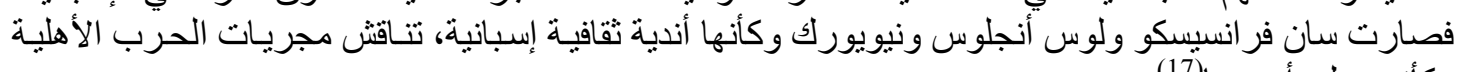

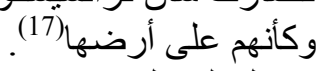

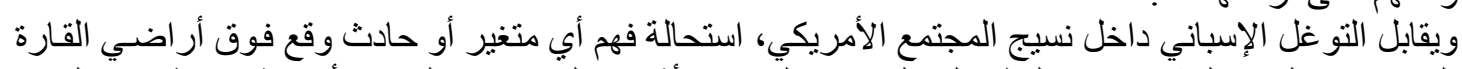

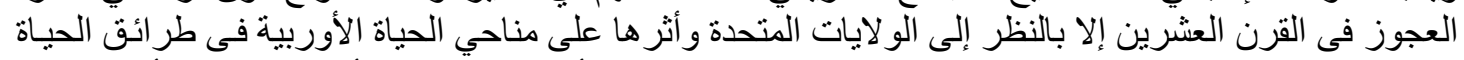

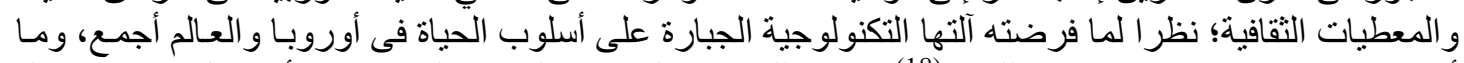

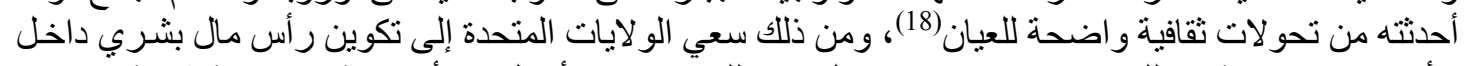

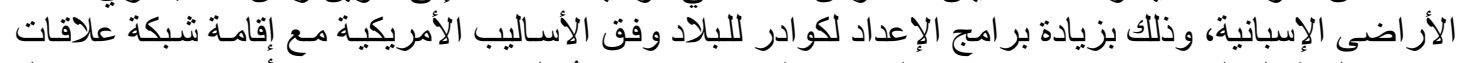

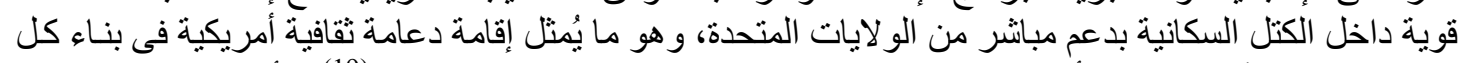

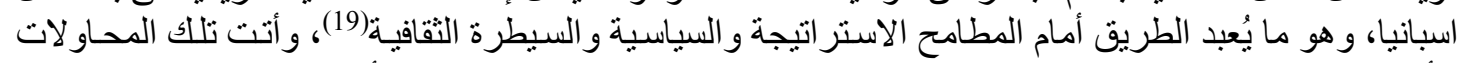

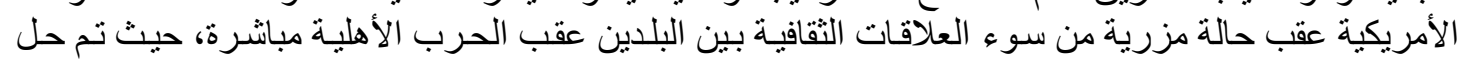

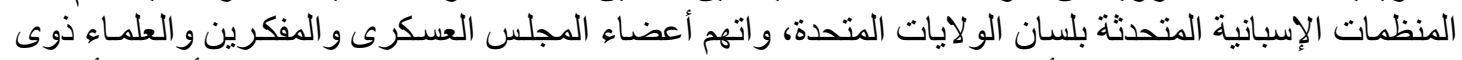

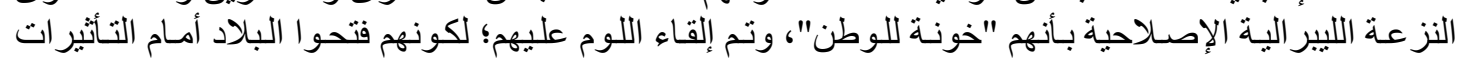

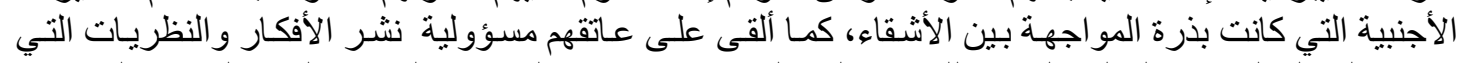

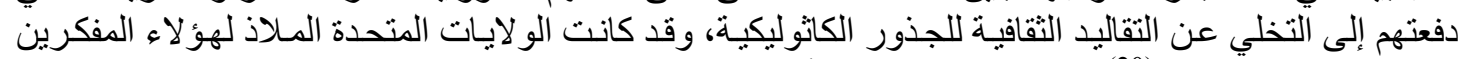

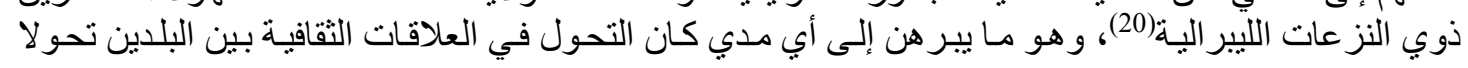




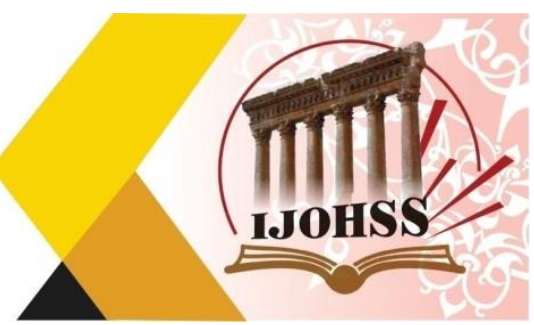

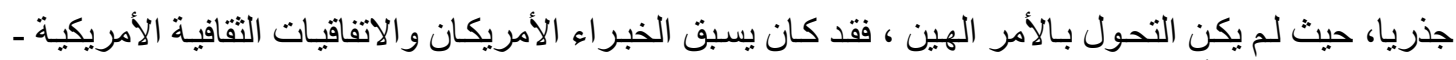

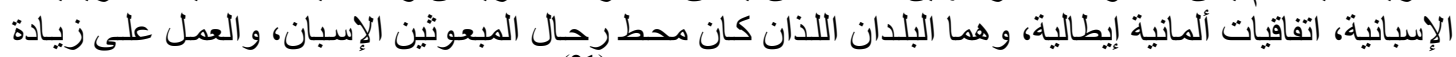

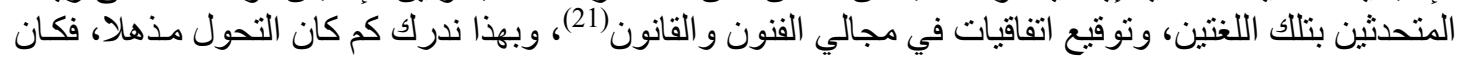

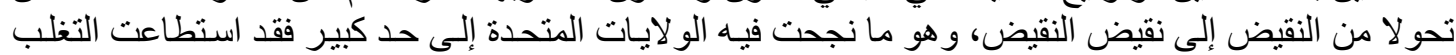

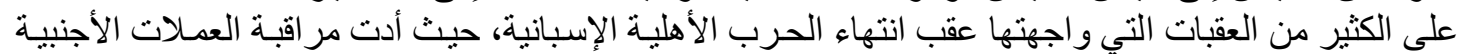

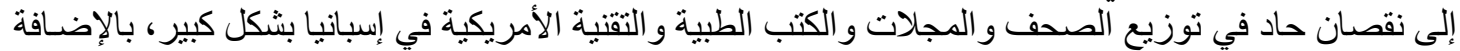

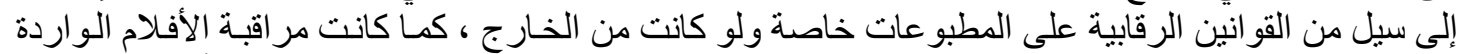

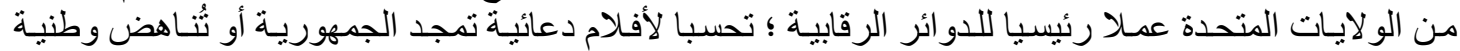

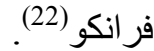

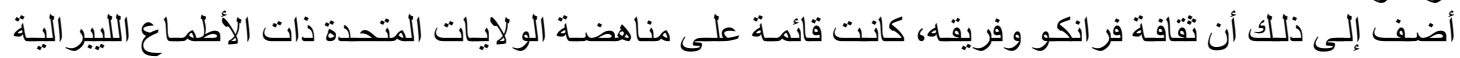

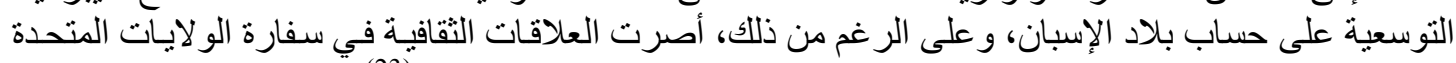

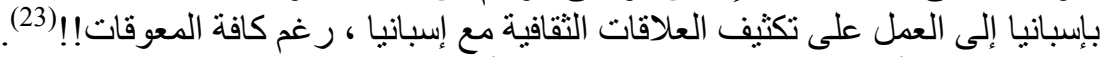

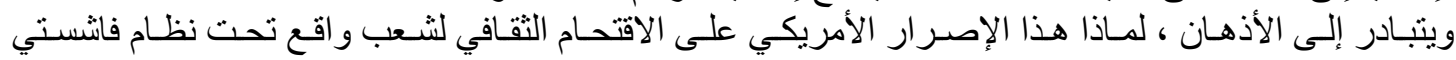

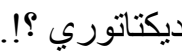

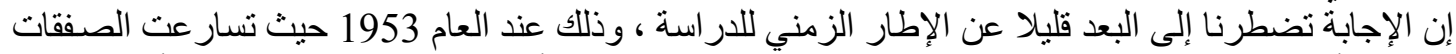

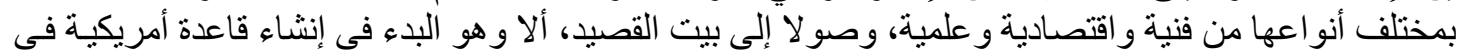

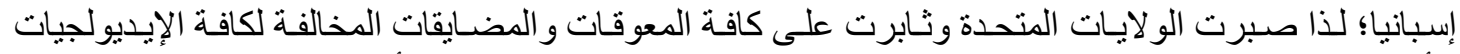

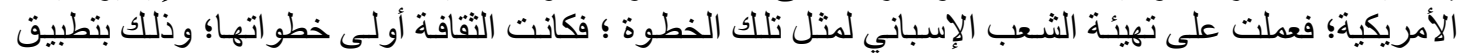

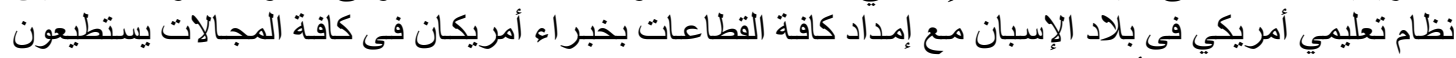

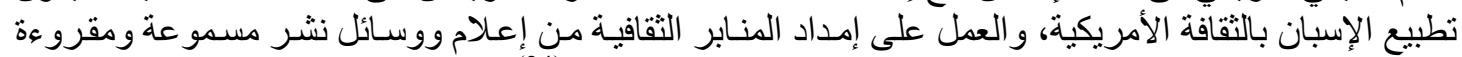

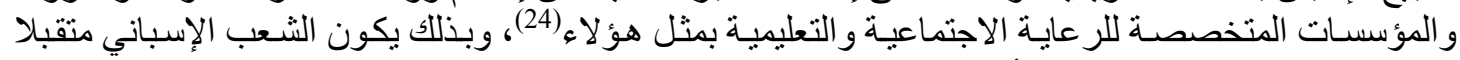

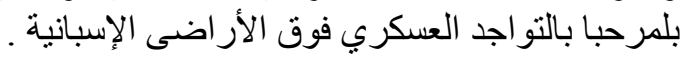

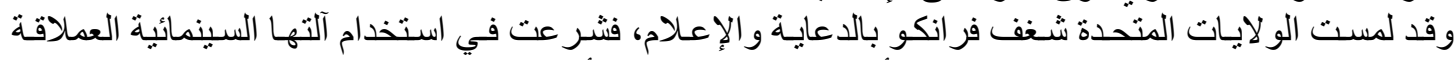

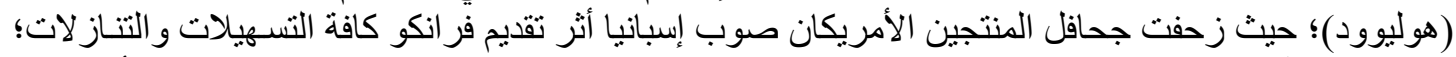

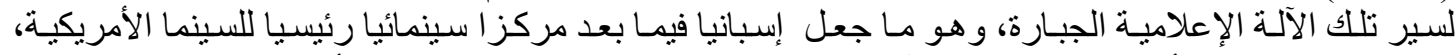

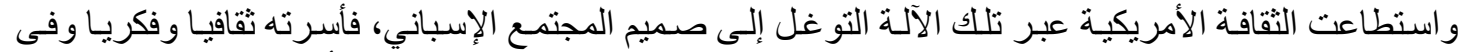

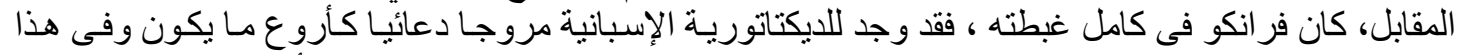

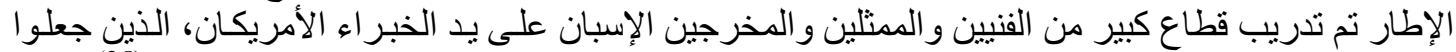

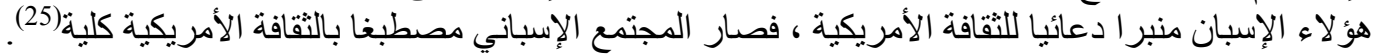

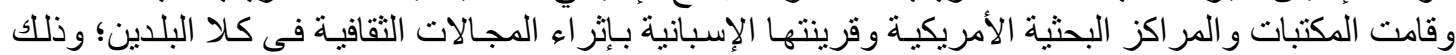

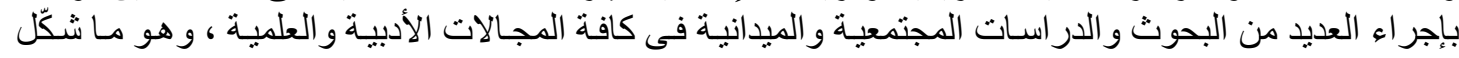

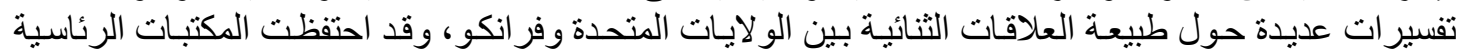

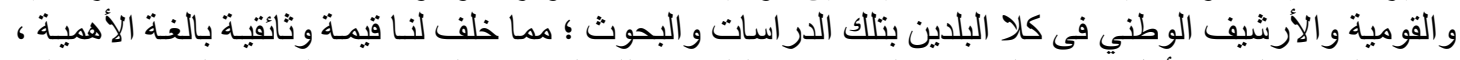

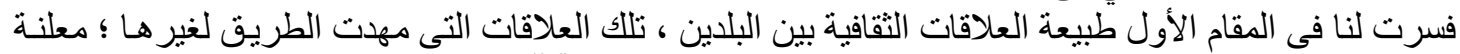
بدء العلاقات ومجالات الثر اكة بين الو لايات المتحات المدة و إسبانية فر انكو (26). 


\section{العلاقات الاقتصادية بين الولايات المتحدة الامريكية واسباتيا}

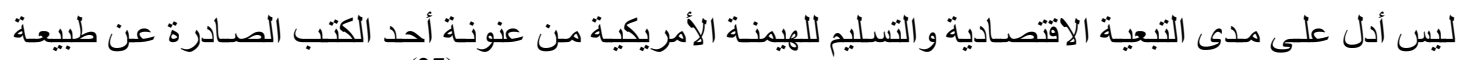

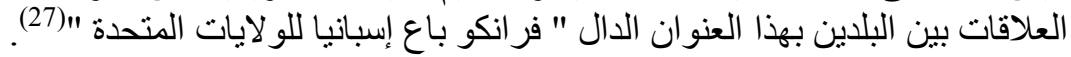

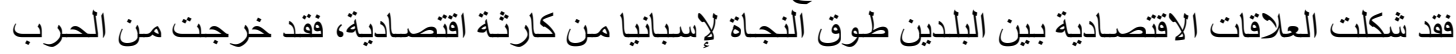

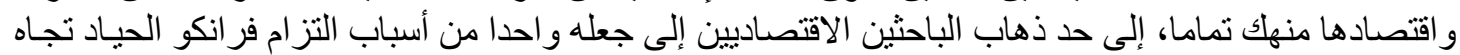

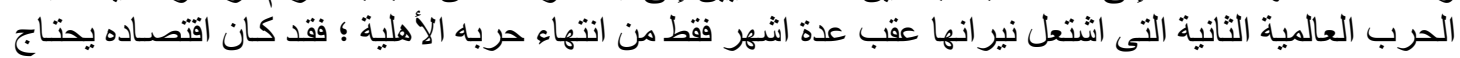

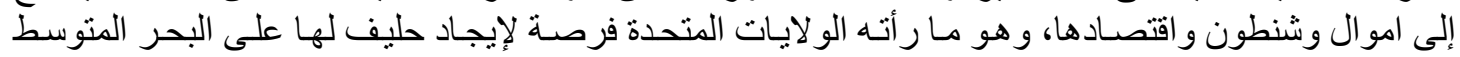

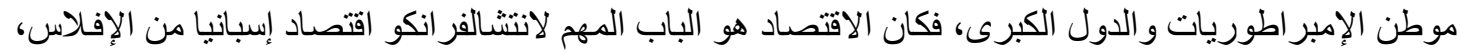

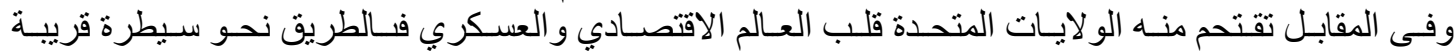

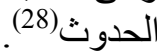

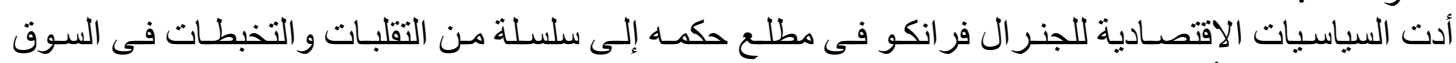

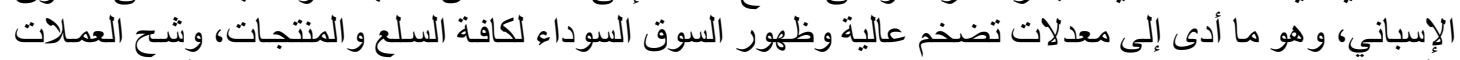

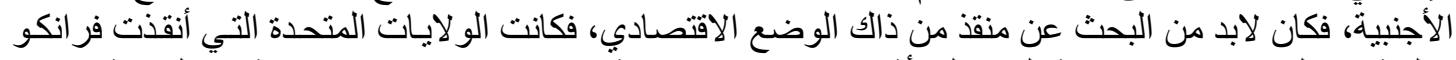

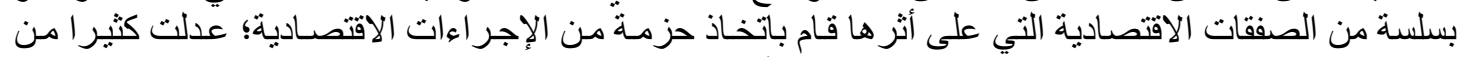

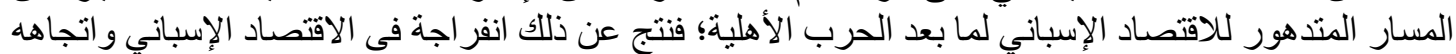

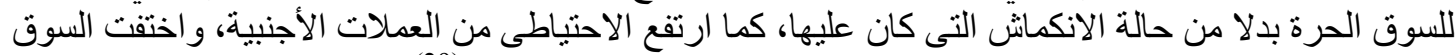

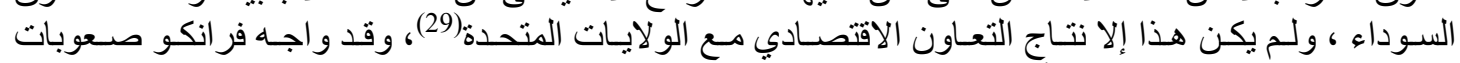

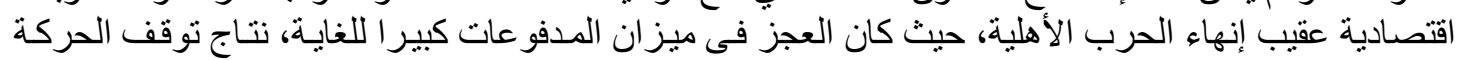

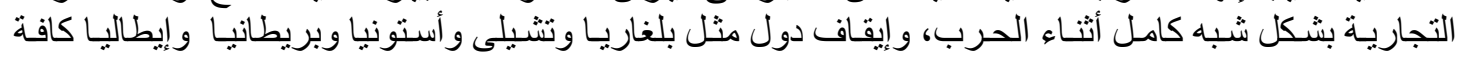

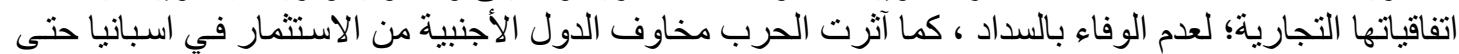

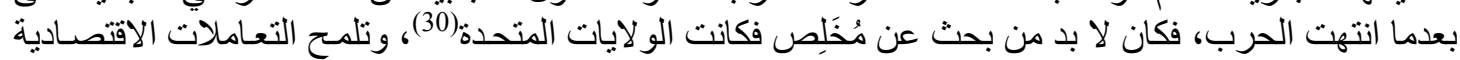

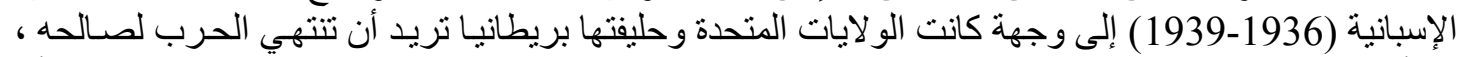

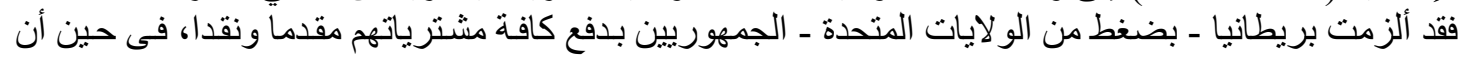

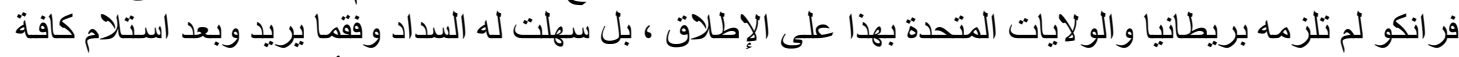

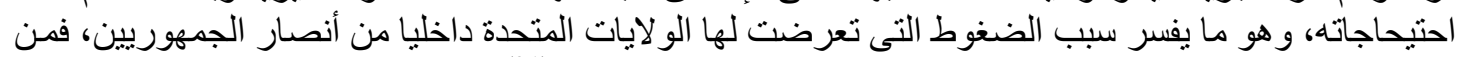

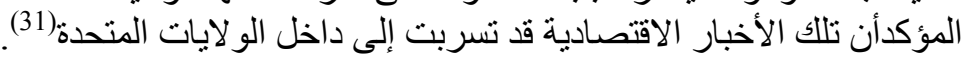

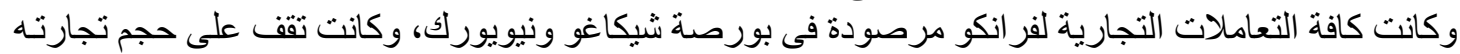

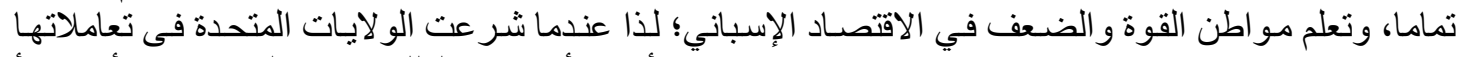

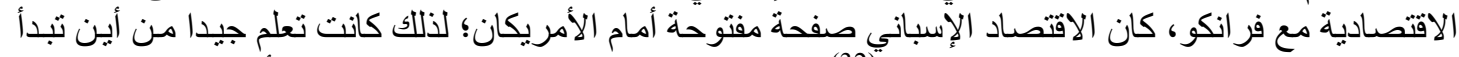

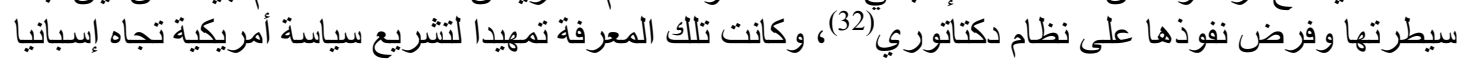

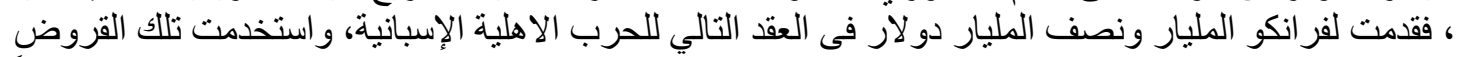

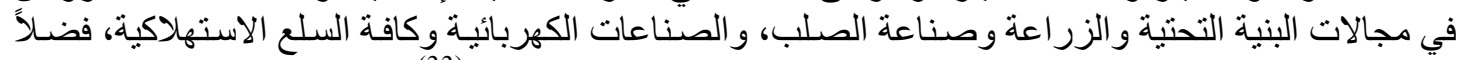

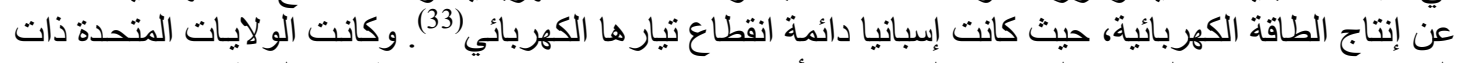

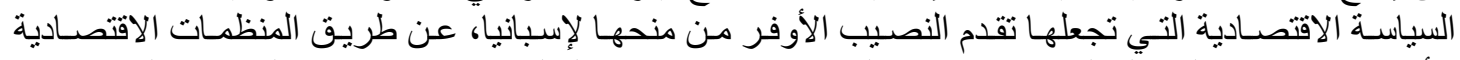

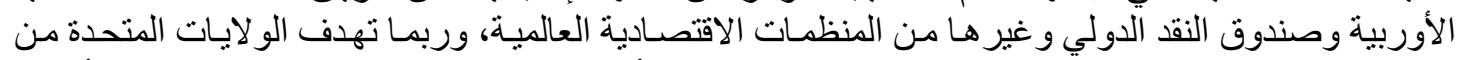

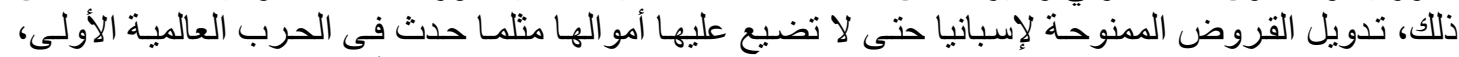

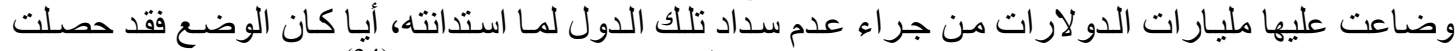

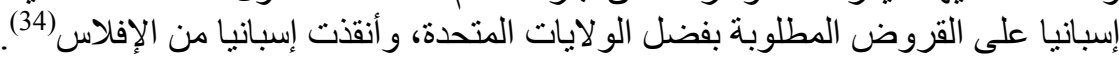

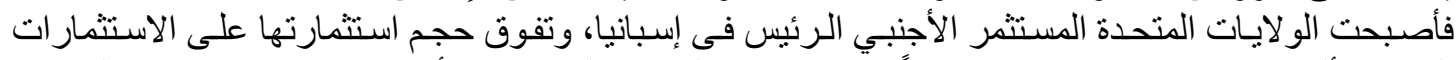

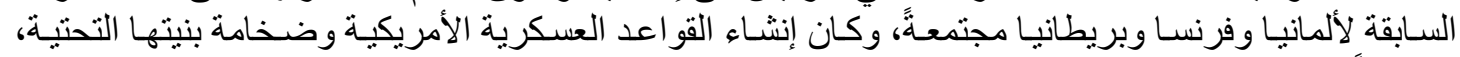

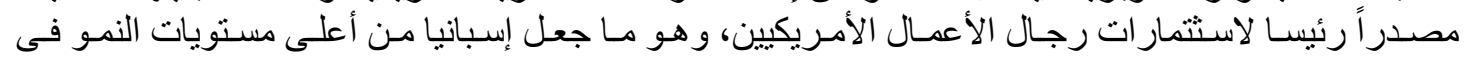




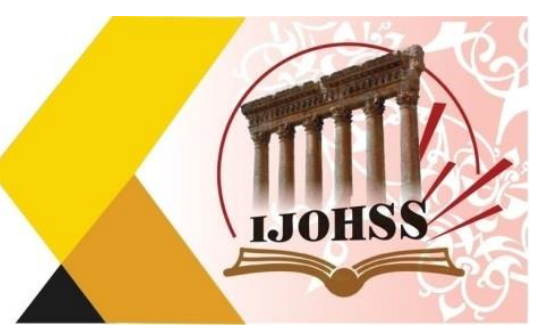

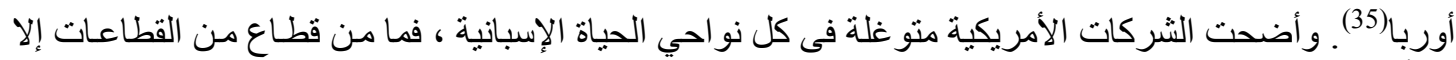

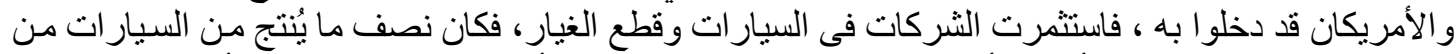

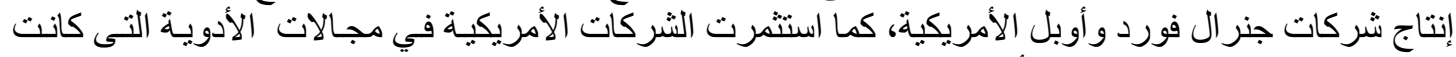

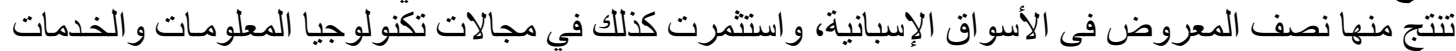

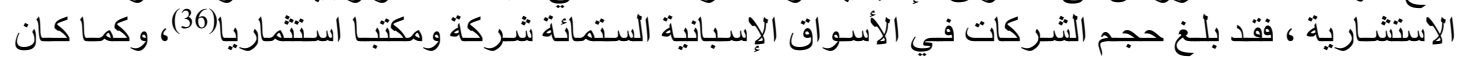

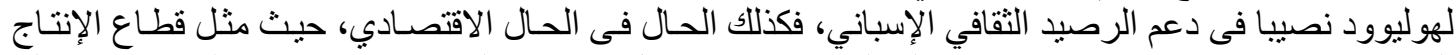

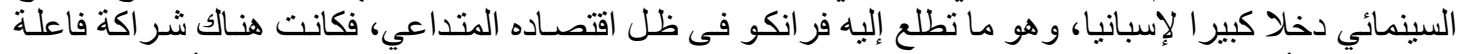

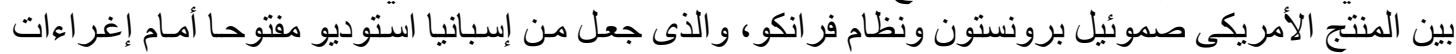

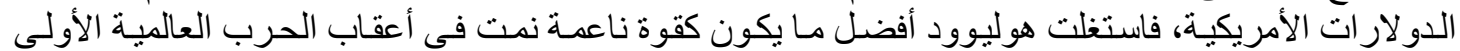

.37) 1918-1914

فأضحت إسبانيا سوقار رائجا أمام المنتجين المستقلين الأمريكان، مستفدين من رخص التهائ التكاليف النسبي بين إسبانيا

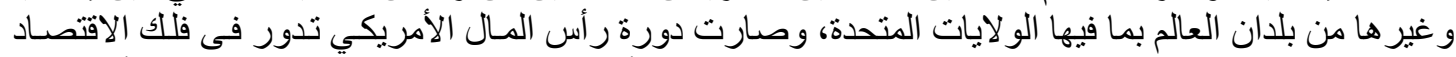

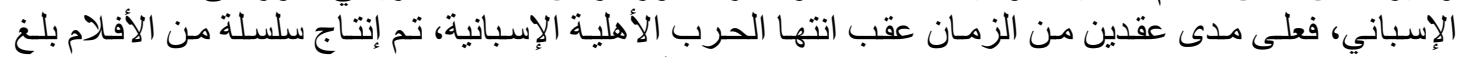

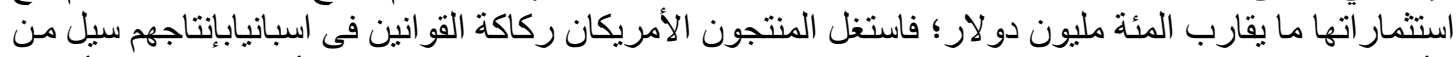

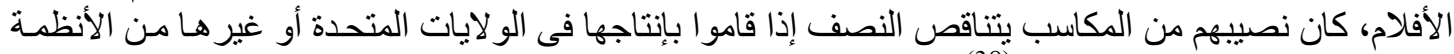
المحكومة بقو انين يصعب اختون الصتر اقهاب(38).

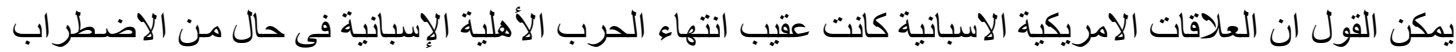

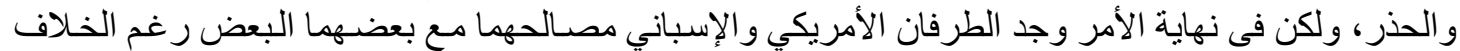

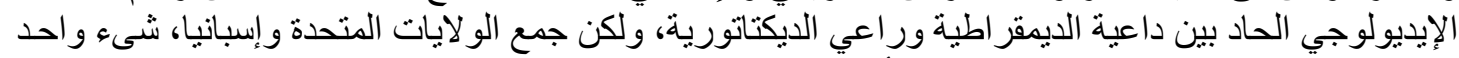

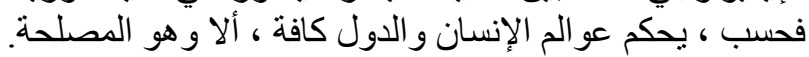

الخاتمة

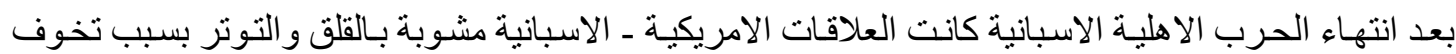

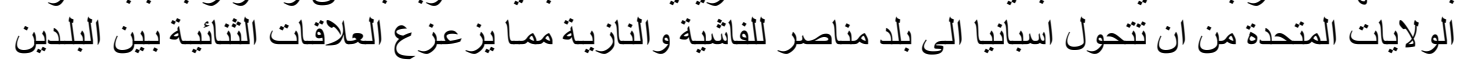

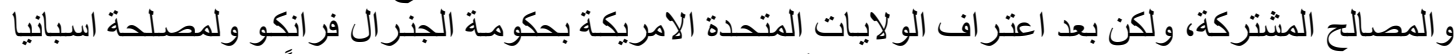

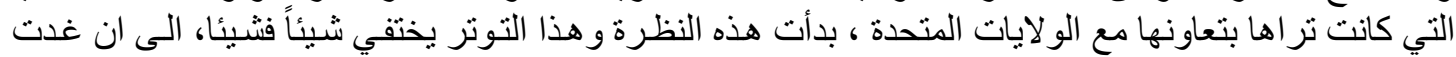

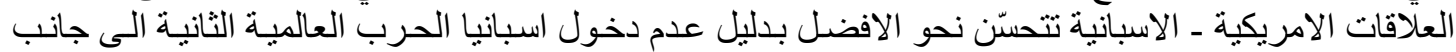

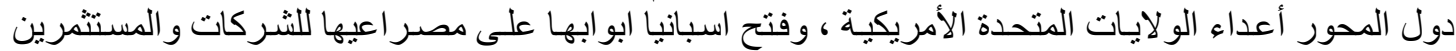
الامريكيين و هذا ادى بالتالي الى نشو الاء علاقة قوية ومتينة بين الجانبين.

\section{الهوامش والمصادر}

1. F.R.U.S, Vol.II ,the secretary of state of the Spanish Minister for Foreign Affairs (Jordana),April 1,1939,p 741 ;Sebastian ,Balfour \& Paul, Preston :Spain and the Great Powers in the Twentieth Century, Rout ledge, London and New York,1999 ,p.229.

2. خوان ديكارديناس (1881-1966): كان سفير اً لإسبانيا في و اشنطن أثناء الجمهورية الثانية بين عامي 1932

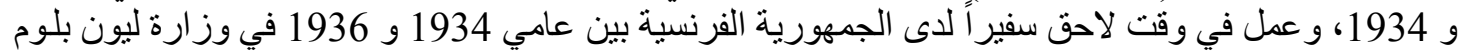

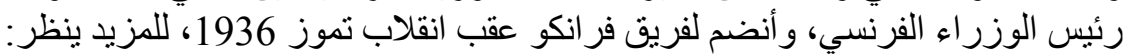
Miralles, Ricardo : diplomáticos y Guerra Civil , Marcial Pons Ediciones de Historia, Madrid, 2010,p.125.

3. VarelaLago, Ana Maria: Conquerors, immigrants, exiles,p.271.

4. New York Times, 1 April 1939,p. 2.

5. New York Times, 5 April 1939,p. 12. 


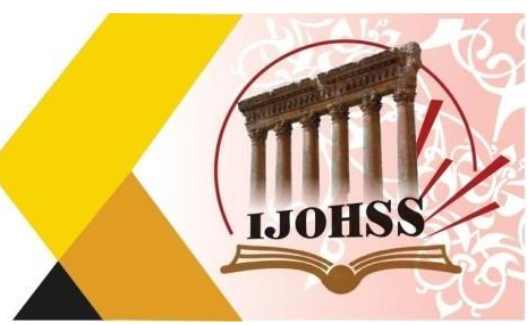

6. ARTURO JARQUE, IÑIGUEZ: ESPAÑA ESTADOS UNIDOS, GUERRA FRÍA Y BASES, Universidad de Alcalá de Henares,p.92.

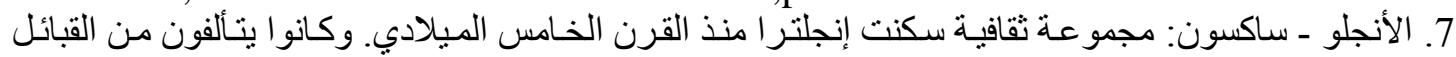

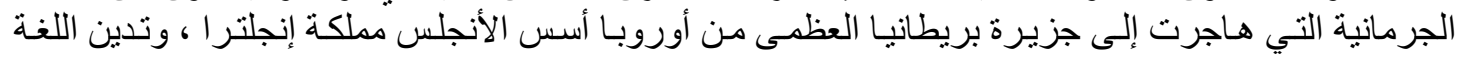

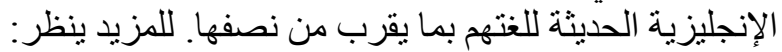

Higham, Nicholas J., and Martin J. Ryan. The Anglo-Saxon World. Yale University Press, 2013. p. 7.

8. HUMPHREYS, R.A: Latin America and the Second World War. Vol. I,19391942. Athlone Press, London, 1981.p.15.

9. Espacio, Tiempo y Forma, Serie V, H." Contemporánea, t. V, 1992,p.260.

10. Ibd,p.261.

11. ARTURO JARQUE, INIGUEZ: ESPAÑA ESTADOS UNIDOS, p.93.

12. ARTURO JARQUE, Op.Cit, p.94.

13. Greenhalgh, Paul: Ephemeral Vistas: The Expositions Universelles, Great Exhibitions and World's Fairs, 1851-1939. Manchester, UK: Manchester University Press, 1988, p. 101.

14. David J. Dunthorn: Spain in an International Context, 1936-1959 , Berghahn Books ,Oxford \& New York, 1999, p. 210.

15. David J. Weber: The Spanish Legacy in North America and the Historical Imagination, the Western Historical Quarterly, Vol. 23, No. 1, (Feb., 1992), p.4.

16. José Antonio Alonso: THE FUTURE OF SPANISH IN THE UNITED STATES: THE LANGUAGE OF HISPANIC MIGRANT COMMUNITIES, Ariel, Barcelona, 2014, p.49.

17. Carlos Nava: INTERNATIONALISM IN THE BARRIOS: HISPANICAMERICANS AND THE SPANISH CIVIL WAR (1936-1939), Dedman College Southern Methodist University, U.S.A, 2020, p. 3.

18. Lorenzo DELGADO: EL AMIGO AMERICANO, ESPAÑA Y ESTADOS UNIDOS DURANTE EL FRANQUISMO, Instituto de Historia-CSIC, 2003, p. 231.

19. Cuadernos de Historia Contemporánea, 25, 2003, p. 35.

20. Antonio NIÑO: 50 años de relaciones entre España y Estados Unidos50 years of Spain-United States relations, Cuadernos de Historia Contemporaneous, 2003,P.36.

21. ás datos sobre ese conjunto de actividades en Delgado Gómez-Escalonilla, Lorenzo: Imperio de papel. Acción cultural y política exterior durante el primer franquismo, Madrid, CSIC, 1992, p. 193.

22. Antonio NIÑO,Op.Cit, P.38.

23. Urpinell, Lluís:Artur Cuyàs: Un polifacètic incansable. IV Jornades d'Estudis Catalano-americans: Generalitat de Catalunya, Barcelona, 1992, P.453.

24. Lorenzo DELGADO,Op.Cit, P.264.

25. Joseph S. Nye \&John D. Donahue: Governance in a Globalizing World, Brookings Institution Press, Washington, 2000, p. 118-119.

26. Pablo leóN aguiNaga : La historiografía española y las relaciones con los Estados Unidos de América: las consecuencias del Pacto de Madrid y laTransición,Cuadernos de Historia Contemporánea, vol. 34, 2012,P.358-359. 


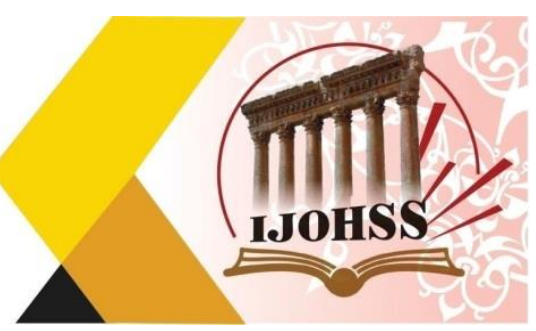

27. Neal M. Rosendorf: Franco Sells Spain to America, paigrave macmillan, U.K, 2014.p30 .

28. William Chislett ,Spain and the United States ,P.15.

29. Leandro Prados de la Escosura: Economic Reforms and Growth in Franco's Spain,Universidad Carlos III,2011,P.2

30. Elena Martínez Ruiz: GUERRA CIVIL, COMERCIO Y CAPITAL EXTRANJERO EL SECTOR EXTERIOR.

31. MORADIELLOS, E: Neutralidad benévola, El Gobierno británico y la insurrección 119ilitary española de 1936, Pentalfa Ediciones, Oviedo,1990 , p. 301303.

32. Los datos sobre exportaciones de jerez se encuentran en Barciela, GEHR, Giráldez y López ,2006, p. 126

33. William Chislett :Spain and the United States ,P.21.

34. Mis conversaciones privadas con Franco , Salgado Araujo Francisco Franco, Planeta, 1976,P. 294.

35. Julio Tascón: International Capital before Capital Internationalisation in Spain, 1936-59, Minda de Gunzburg Centre for European Studies, Harvard University, Working Paper, No.79, P.15.

36. William Chislett,op.cit,P.47.

37. Neal M. Rosendorf: Franco Sells Spain to America, p.49.

38. Joseph S. Nye \&John D. Donahue: Governance in a Globalizing World,p.119. 